msh-mss Mathématiques et sciences humaines

191 | Automne 2010

Variabilité et inégalités

\title{
Mesure de la dispersion et des inégalités. Quelques idées
}

\section{Marc Barbut}

\section{(2) OpenEdition}

\section{Journals}

Édition électronique

URL : http://journals.openedition.org/msh/11883

DOI : $10.4000 / \mathrm{msh} .11883$

ISSN : 1950-6821

Éditeur

Centre d'analyse et de mathématique sociales de l'EHESS

\section{Édition imprimée}

Date de publication : 1 septembre 2010

Pagination : 7-32

ISSN : 0987-6936

\section{Référence électronique}

Marc Barbut, " Mesure de la dispersion et des inégalités. Quelques idées », Mathématiques et sciences

humaines [En ligne], 191 | Automne 2010, mis en ligne le 15 février 2011, consulté le 23 juillet 2020.

URL : http://journals.openedition.org/msh/11883 ; DOI : https://doi.org/10.4000/msh.11883 


\title{
MESURES DE LA DISPERSION ET DES INÉGALITÉS. QUELQUES IDÉES
}

\author{
Marc BARBUT ${ }^{1}$
}

RÉSUMÉ - On présente sommairement cinq façons bien différentes dans leur esprit et dans leur mode opératoire d'appréhender la notion de dispersion (concentration) d'une distribution statistique ou de probabilité, dans le seul cas univarié. On met l'accent sur des mesures fonctionnelles de la dispersion. Dans un dernier paragraphe, on évoque le cas de la mesure des inégalités.

MOTS-CLÉS - Concentration, Concentration-dispersion (fonctions de), Diamètre, Dispersion, Écart, Inégalités, Quantiles

SUMMARY - Dispersion and inequality measures: six ideas

This paper is a brief introduction to five very different ways to define the notion of dispersion (concentration) in an empirical or probability distribution, in the univariate case. We emphasize the approach by functional measures of dispersion. The last section is about the functional measures of inequality.

KEYWORDS - Concentration, Concentration (dispersion) functions, Deviation, Diameter, Dispersion, Inequality, Quantiles

\section{INTRODUCTION}

En juin 1989, d'éminents chercheurs en sciences sociales avaient organisé à Paris un colloque intitulé «Moyenne, milieu, centre» (cf. Armatte [1991]). C'était, selon la doctrine d'Adolphe Quetelet, témoigner du rôle essentiel, pour l'analyse statistique des données, des valeurs centrales.

Mais pour une distribution, une même valeur centrale peut correspondre à des situations radicalement différentes, notamment en ce qui concerne la dispersion (ou la concentration) autour (attention : ce n'est même pas toujours « autour »!) de ce centre.

Il convenait donc de compléter l'initiative de 1989 par une rencontre consacrée à la dispersion, la variabilité et l'inégalité. Ce fut fait à la Sorbonne en novembre 2009 et c'est un exposé qui y fut présenté en introduction aux notions de dispersion (concentration) en statistique que l'on trouve ci-dessous.

D'autres interventions à ce colloque seront publiées dans Mathématiques et Sciences humaines ou encore dans le Journal Électronique d'Histoire de la Probabilité et de la Statistique (jehps.net), livraison de juin 2010.

\footnotetext{
${ }^{1}$ Centre d'analyse et de mathématique sociales (CAMS), École des hautes études en sciences sociales, 190-198 avenue de France 75244 Paris cedex 13, mbarbut@ehess.fr
} 
Sans qu'il y ait rien d'original, on y insiste sur cinq idées concernant cinq façons différentes d'appréhender la notion de dispersion et de la rendre opératoire. Une sixième section consiste en l'application des plus fécondes des précédentes, celles des mesures fonctionnelles de dispersion, à la mesure des inégalités. C'est la sixième idée.

Les deux premiers paragraphes concernent les deux plus usuelles des mesures de la dispersion d'une distribution par un seul nombre, tels que le plus célèbre d'entre eux, la variance.

Mais une même variance (ou une même valeur de tel indicateur numérique de la concentration) peut, comme une même valeur du centre, correspondre à son tour à des situations très différentes.

Pour pallier cet inconvénient, une solution « à la Karl Pearson » consiste à ajouter des moments de la distribution, ceux d'ordre 3 et 4 le plus souvent. Mais où s'arrêter ?

Une autre solution, celle empruntée de 1906 à 1930 environ par les M.O. Lorenz, C. Gini, P. Lévy, M. Fréchet, E. Gumbel, et même, en un certain sens, par G. Sorel dès 1897 : associer à chaque distribution une fonction de la variable en jeu, fonction censée représenter au mieux sa dispersion (ou son inégalité).

Bien sûr, il en résulte que, dans la comparaison, on n'aura plus un ordre total (comme c'est le cas pour un indicateur numérique); en général, l'ordre ne sera que partiel. Mais n'est-ce pas justement là dans la nature des choses ? D'ailleurs, le calcul de plusieurs moments conduit lui aussi à un ordre partiel.

Deux avertissements pour terminer :

- On n'aborde pas ici, sauf par quelques très sommaires et très partielles indications, la question des indices (ou coefficients) d'inégalité ; la dispersion est le sujet central ;

- Dans ce texte élémentaire on reste dans le cadre le plus simple, celui de la statistique uni-variée. L'extension au cas multi-varié de certaines des notions présentées ici pose de délicats problèmes. Il faudrait y consacrer tout un autre colloque.

\section{PREMIÈRE IDÉE : UN CENTRE ET UN ÉCART AU CENTRE}

C'est l'idée la plus banale, la plus communément mise en pratique. Le lecteur qui en est familier peut donc sauter ce paragraphe.

\subsection{DISTANCES}

Soient deux suites d'observations de variables numériques portant sur le même ensemble de $n$ individus (ce que l'on appelle deux variables appariées) :

$$
x=\left(x_{1}, x_{2}, \ldots, x_{i}, \ldots, x_{n}\right) \quad y=\left(y_{1}, y_{2}, \ldots, y_{i}, \ldots, y_{n}\right)
$$

En quoi diffèrent-elles?

Première réponse, la plus immédiate: par leurs différences $\left(x_{i}-y_{i}\right)$, c'est-à-dire la suite :

$$
x-y=\left(x_{1}-y_{1}, \ldots, x_{i}-y_{i}, \ldots, x_{n}-y_{n}\right)
$$


Problème : résumer cette suite de $n$ différences individuelles par un seul nombre.

La solution : associer à chaque couple de suites $x$ et $y$ un nombre positif $\Delta(x, y)$, leur distance, vérifiant (quels que soient $x, y$ et $z$ ) les trois conditions :

$$
\begin{aligned}
& \Delta(x, y)=0 \quad \text { ssi } \quad x=y \\
& \Delta(x, y)=\Delta(y, x) \quad \text { (symétrie) } \\
& \Delta(x, z) \leq \Delta(x, y)+\Delta(y, z) \quad \text { (inégalité triangulaire) }
\end{aligned}
$$

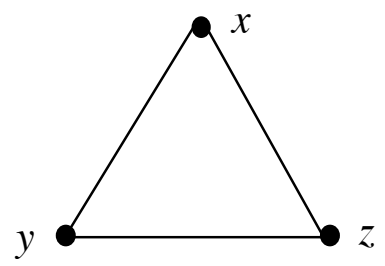

\subsection{UNE FAMILLE DE DISTANCES}

On démontre, on ne le fera pas ici (voir par exemple, Barbut [1986(a]) que, quel que soit l'exposant $\alpha \geq 1, \Delta_{\alpha}(x, y)$ défini par l'équation (1) est une distance :

$$
\Delta_{\alpha}(x, y)=\left(\sum_{i=1}^{n}\left|x_{i}-y_{i}\right|^{\alpha}\right)^{1 / \alpha} \quad \alpha \geq 1
$$

En pratique, seules trois valeurs de l'exposant $\alpha$ sont utilisées.

- $\alpha=1 \quad \Delta_{1}(x, y)=\sum_{i=1}^{n}\left|x_{i}-y_{i}\right|$

Pour la dimension $n=2$, on a : $\Delta_{1}(x, y)=\left|x_{1}-y_{1}\right|+\left|x_{2}-y_{2}\right|$

c'est la valeur commune de tous les « escaliers » reliant les points $x$ et $y$ (cf. Figure 1), et en particulier du chemin $x \alpha y$. D'où le nom de «distance de Manhattan » qu'on lui donne souvent.

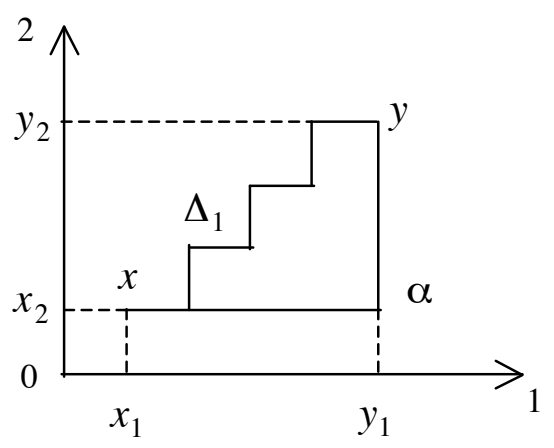

Figure 1

$-\alpha=2 \quad \Delta_{2}(x, y)=\left(\sum_{i=1}^{n}\left(x_{i}-y_{i}\right)^{2}\right)^{1 / 2}$

C'est la classique «distance euclidienne », la longueur de l'hypothénuse du triangle $x a y$ (cf. Figure 2) dans le cas de la dimension $n=2$. 


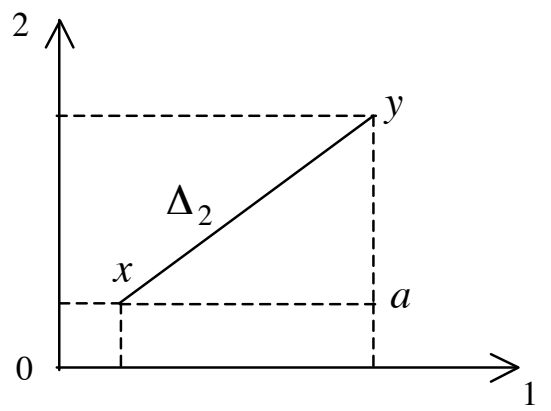

Figure 2

$-\alpha=\infty \quad \Delta_{\infty}(x, y)=\max _{i}\left|x_{i}-y_{i}\right|$

Pour $n=2$ (cf. Figure 3), c'est le plus grand côté de l'angle droit dans le triangle $x$ a y. Cette distance est appelée distance de la convergence uniforme.

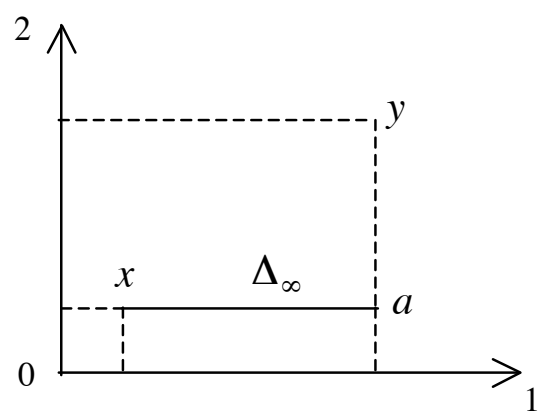

Figure 3

\subsection{CENTRES ET ÉCARTS}

Soit maintenant une suite $x$ de $n$ observations d'une même variable numérique $X$ (par exemple une même variable $X$ observée sur $n$ individus)

$$
x=\left(x_{1}, x_{2}, \ldots, x_{i}, \ldots, x_{n}\right)
$$

Problème : résumer cette suite de $n$ nombres par un seul nombre, qu'on appellera valeur centrale (ou typique), ou plus brièvement centre de la suite des observations.

Une solution :

- considérons une suite de $n$ observations qui seraient constamment égales à une même valeur $\lambda$. On peut la noter $\lambda u$, où $u$ est la suite constante :

$$
u=(1,1, \ldots, 1, \ldots, 1)
$$

ce qui donne :

$$
\lambda u=(\lambda, \lambda, \ldots, \lambda, \ldots \lambda)
$$

- choisissons une distance $\Delta$. Alors si $\lambda$ varie, $\Delta(x, \lambda u)$ est une fonction de $\lambda$ :

$$
\varphi(\lambda)=\Delta(x, \lambda u)
$$

- faisons varier $\lambda$ de $-\infty$ à $+\infty$; en général, $\varphi(\lambda)$ aura un minimum pour $\lambda=c$. 


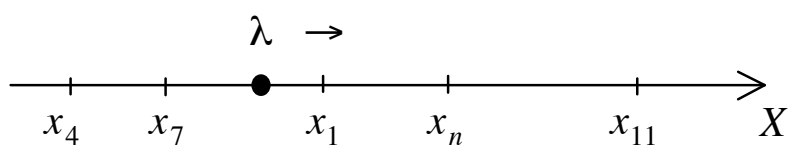

Figure 4

C'est ce minimum $c$, ou plus précisément $c(x)$, car il dépend évidemment de la suite $x$ des observations, que l'on prendra pour centre de celles-ci.

Quant à la distance minimum $\Delta(x, c u)$, on l'appelle écart de la suite $x$ pour la distance $\Delta$. C'est une mesure de la dispersion (ou dualement de la concentration) des observations :

$$
E(x)=\Delta(x, c u)
$$

Exemples :

- Pour la distance $\Delta_{\infty}$ de la convergence uniforme, on a :

$$
\varphi(\lambda)=\Delta_{\infty}(x, \lambda u)=\max _{i}\left|x_{i}-\lambda\right|
$$

Il est clair (cf. Figure 4) que $\varphi(\lambda)$ est minimum pour :

$$
\lambda=\frac{1}{2}\left(\max _{i} x_{i}+\min _{i} x_{i}\right)=c
$$

Le centre est le milieu de l'intervalle séparant la plus petite des observations de la plus grande (cf. Figure 5).

L'écart correspondant est par suite :

$$
E=\frac{1}{2}\left(\max _{i} x_{i}-\min _{i} x_{i}\right)
$$

C'est la moitié de l'intervalle séparant la plus petite des observations de la plus grande.

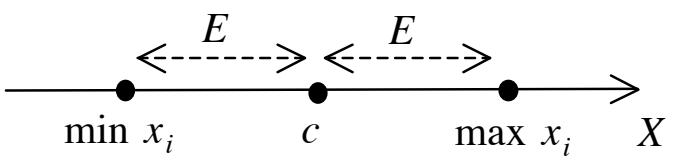

Figure 5

- Pour la distance euclidienne $\Delta_{2}$, on a :

$$
\varphi(\lambda)=\Delta_{2}(x, \lambda u)=\left(\sum\left(x_{i}-\lambda\right)^{2}\right)^{1 / 2}
$$

Il est clair que $\varphi(\lambda)$ et $\varphi^{2}(\lambda)$ ont leur minimum pour la même valeur de $\lambda$. Or :

$$
\varphi^{2}(\lambda)=\sum\left(x_{i}-\lambda\right)^{2}=\sum x_{i}^{2}-2 \lambda \sum x_{i}+n \lambda^{2}
$$

Ce polynôme du second degré est minimum pour : 


$$
\lambda=\frac{1}{n} \sum x_{i}=c
$$

Le centre est la moyenne arithmétique, notée $m$ dans la suite, des observations.

Quant à l'écart, appelé écart quadratique, son carré est :

$$
E^{2}=\sum\left(x_{i}-m\right)^{2}=\left(\sum x_{i}^{2}\right)-n m^{2}
$$

La moyenne $\frac{E^{2}}{n}$ est ainsi la classique variance $V$ :

$$
\frac{1}{n} E^{2}=V=\frac{1}{n}\left(\sum x_{i}^{2}\right)-m^{2}
$$

Ce qui se dit la variance est « la moyenne des carrés moins le carré de la moyenne ».

Quant à l'écart moyen lui-même, c'est l'écart-type :

$$
\sigma=V^{1 / 2}=\frac{E}{n^{1 / 2}}=\left(\frac{1}{n} \sum x_{i}^{2}-m^{2}\right)^{1 / 2}
$$

- Pour la distance de Manhattan $\Delta_{1}$, le centre est la médiane $\mu$ des valeurs $x_{i}$ observées, si celles-ci sont en nombre impair. Si leur nombre est pair, on a un intervalle médian, dont n'importe quel point peut être pris pour centre. On a en effet :

$$
\varphi(\lambda)=\sum\left|x_{i}-\lambda\right|=\sum_{x_{i}<\lambda}\left(\lambda-x_{i}\right)+\sum_{x_{i}>\lambda}\left(x_{i}-\lambda\right)
$$

Lorsque $\lambda$ croît de $-\infty$ à $+\infty$, chacun des deux termes décroît (cf. Figure 4 supra) tant qu'il y a moins de $x_{i}$ inférieurs à $\lambda$ que de $x_{i}$ qui lui sont supérieurs; et croît lorsque c'est l'inverse. Le minimum est donc atteint lorsqu'il y a autant de $x_{i}$ de part et d'autre de $\lambda$.

L'écart correspondant, dit écart absolu, est alors :

$$
E=\varphi(\mu)=\sum_{x_{i}>\mu} x_{i}-\sum_{x_{i}<\mu} x_{i}
$$

L'interprétation de cette valeur de l'écart est extrêmement claire si les $x_{i}$ sont par exemple des revenus. Convenons d'appeler « riches » ceux qui ont un revenu supérieur au revenu médian, et « pauvres » ceux dont le revenu est inférieur à $\mu$.

L'écart $E$ se lit alors « revenu global des riches moins revenu global des pauvres ».

Le rapport $\frac{E}{n}$ est appelé écart moyen absolu.

NB.

a. Il arrive, chez les économistes notamment, que l'on calcule l'écart absolu à la moyenne $m$ :

$$
\varphi(m)=\sum\left|x_{i}-m\right|
$$


Pourquoi pas ? mais on n'a plus alors l'interprétation très parlante de l'écart à la médiane.

Supposons en effet $n$ impair, soit : $n=2 k+1$. Si les $x_{i}$ sont indiciés dans l'ordre croissant :

$$
x_{1} \leq x_{2} \leq \ldots \leq x_{k+1} \leq \ldots \leq x_{2 k+1}
$$

et si: $x_{h}<m \leq x_{h+1}$, on a

$$
\sum\left|x_{i}-m\right|=\sum_{x_{i}>m} x_{i}-\sum_{x_{i}<m} x_{i}+2(h-k) m
$$

Règle d'or. À la métrique euclidienne $\Delta_{2}$ correspondent la moyenne $m$ et l'écarttype. À la métrique « de Manhattan » $\Delta_{1}$, la médiane $\mu$ et l'écart moyen absolu par rapport à $\mu$.

b. On utilise parfois comme valeur centrale d'une distribution le mode $M$ de celle-ci, c'est-à-dire la valeur la plus fréquente, également appelée dominante.

C'est, par exemple le cas en démographie, où l'âge auquel la mortalité est maximum est d'ailleurs appelé «vie normale », à bien distinguer de la «vie moyenne » (espérance de vie) et de la « vie probable » (médiane des durées de vie).

L'une des propriétés que l'on doit exiger d'une «valeur centrale», supposée représentative de l'ensemble des observations $x_{i}$, est que si tous les $x_{i}$ croissent, la valeur censée les représenter croisse elle aussi.

Or, pour le mode, il y a des cas où c'est le contraire qui se produit : les $x_{i}$ croissent $\left(\forall i, x_{i}>x_{i}^{\prime}\right)$ et le mode $M$ diminue $\left(M<M^{\prime}\right)$.

Voici un exemple très simple de ce phénomène.

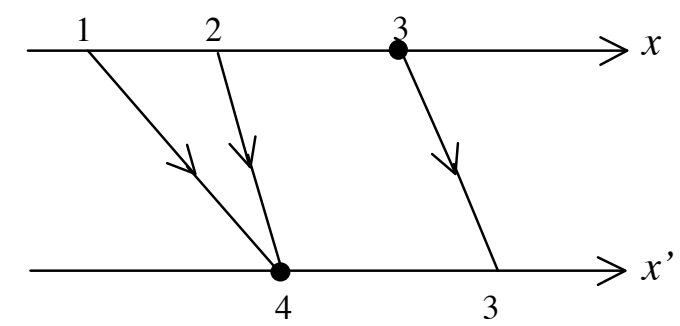

$$
\begin{array}{lll}
x_{1}=x_{2}=0 \quad x_{3}=x_{4}=1 & x_{5}=x_{6}=x_{7}=5 & M=5 \text { (fréquence 3) } \\
x_{1}^{\prime}=x_{2}=x_{3}^{\prime}=x_{4}^{\prime}=2 & x^{\prime}{ }_{5}=x_{6}^{\prime}{ }_{6}=x_{7}^{\prime}=6 & M^{\prime}=2 \text { (fréquence 4) }
\end{array}
$$

Figure 6

\subsection{INDICES D’INÉGALITÉ ASSOCIÉS}

Les écarts sont tous homogènes à la variable $X$ : ils sont de dimension 1 par rapport à celle-ci, et s'expriment dans la même unité de mesure. 
On transforme chacun en un indice (ou indicateur, ou coefficient) d'inégalité de la distribution des observations en le divisant par une grandeur le ramenant à la dimension 0 .

C'est ainsi que l'écart absolu peut être divisé par la somme $S$ des $x_{i}$ :

$$
\frac{E}{S}=\frac{1}{S} \sum_{x_{i}>\mu} \mathrm{x}_{\mathrm{i}}-\frac{1}{S} \sum_{x_{i}<\mu} x_{i}, \quad S=\sum x_{i}
$$

Ce qui s'interprète comme la proportion du revenu global attribuée aux « riches » diminuée de celle qui est attribuée aux «pauvres ».

De même, à l'écart-type correspond, en le divisant par la moyenne, le coefficient de variation $C$ :

$$
C=\frac{\sigma}{m}=\left(\frac{V}{m^{2}}\right)^{1 / 2}
$$

Dans le cas de la distance $\Delta_{\infty}$, on peut considérer le rapport qui, à ma connaissance, n'a pas de nom :

$$
\frac{\max x_{i}-\min x_{i}}{\max x_{i}+\min x_{i}}
$$

NB. Les trois indices d'inégalité ainsi définis n'ont vraiment de sens que pour les variables positives. Sinon, la somme ou la moyenne des $x_{i}$ peuvent être proches, voire très proches de 0 ; ou négatives: le rapport calculé n'a plus aucun sens en termes d'inégalité.

\section{DEUXIÈME IDÉE : UN DIAMÈTRE}

Ici, il n'y a plus trace de centre, ni de valeur représentative des $x_{i}$. A. Quetelet n'aurait pas aimé.

\subsection{DIAMÈTRE D'UNE DISTRIBUTION}

Notons toujours $x=\left(x_{1}, x_{2}, \ldots, x_{n}\right)$ la suite des valeurs observées.

L'idée est de considérer l'ensemble des différences $\left|x_{i}-x_{j}\right|$. On posera donc, pour tout $\alpha \geq 1$ :

$$
D_{\alpha}(x)=\left[\sum_{i=j}^{n} \sum_{j=1}^{n}\left|x_{i}-x_{j}\right|^{\alpha}\right]^{1 / \alpha}=\left[2 \sum_{x_{i}>x_{j}} \sum_{i}\left(x_{i}-x_{j}\right)^{\alpha}\right]^{1 / \alpha}
$$

$D_{\alpha}$ est le diamètre d'ordre $\alpha$.

Pour définir un diamètre moyen, on peut considérer soit le nombre total $n^{2}$ des couples $\left(x_{i}, x_{j}\right)$, soit celui des $n(n-1)$ couples tels que $i \neq j$; ce qui conduit aux diamètres moyens : 


$$
\begin{aligned}
& -\quad d_{\alpha}(x)=\left[\frac{1}{n^{2}} \sum_{1}^{n} \sum_{1}^{\mathrm{n}}\left|\mathrm{x}_{\mathrm{i}}-x_{j}\right|^{\alpha}\right]^{1 / \alpha} \\
& -\quad \delta_{\alpha}(x)=\left[\frac{1}{n(n-1)} \sum_{i} \sum_{j}\left|\mathrm{x}_{\mathrm{i}}-x_{j}\right|^{\alpha}\right]^{1 / \alpha}
\end{aligned}
$$

On a évidemment :

$$
\delta_{\alpha}=\left(\frac{n}{n-1}\right)^{1 / \alpha} d_{\alpha}, \quad \alpha \geq 1
$$

Quand le nombre $n$ des observations est assez grand, ces deux diamètres moyens diffèrent peu l'un de l'autre.

Dans le cas $\alpha=1$ :

$$
\delta_{1}=\frac{1}{n(n-1)} \sum_{i} \sum_{\mathrm{j}}\left|x_{i}-x_{j}\right|=\frac{2}{n(n-1)} \sum_{x_{i}>x_{j}} \sum_{i}\left(x_{i}-x_{j}\right)
$$

En divisant $\delta_{1}$ par le total $\left(\sum x_{i}\right)$ distribué, nous obtenons l'indice d'inégalité $G$ de Gini :

$$
\text { (3) } \quad G=\frac{\delta_{1}}{\sum x_{i}}
$$

Dans le cas $\alpha=2$, on a :

$$
\begin{aligned}
\left(D_{2}(x)\right)^{2}= & \sum_{i=1}^{n} \sum_{j=1}^{n}\left(x_{i}-x_{j}\right)^{2}=\sum \sum\left(x_{i}-2 x_{i} x_{j}+x_{j}\right)^{2} \\
& =n \sum x_{i}{ }^{2}-2 \sum \sum x_{i} x_{j}+n \sum x_{j}{ }^{2}
\end{aligned}
$$

Or :

$$
\sum_{i} \sum_{j} x_{i} x_{j}=\left(\sum_{1}^{n} x_{i}\right)\left(\sum_{1}^{n} x_{j}\right)=\left(\sum x_{i}\right)^{2}
$$

D'où finalement :

$$
\begin{aligned}
\left(D_{2}(x)\right)^{2} & =2\left[n \sum x_{i}^{2}-\left(\sum x_{i}\right)^{2}\right] \\
& =2 n^{2}\left[\frac{1}{n} \sum x_{i}^{2}-\left(\frac{\sum x_{i}}{n}\right)^{2}\right] \\
& =2 n^{2} V
\end{aligned}
$$

D'où, pour le diamètre moyen $d_{2}$ :

$$
d_{2}(x)=\left[\frac{1}{n^{2}} \sum \sum\left(x_{i}-x_{j}\right)^{2}\right]^{1 / 2}
$$




$$
d_{2}(x)=(2 V)^{1 / 2}=\sqrt{2} \sigma
$$

- Dans le cas où $\alpha=\infty$, on obtient, par passage à la limite quand $\alpha$ tend vers l'infini :

$$
D_{\infty}(x)=2 E_{\infty}(x)=\max _{i} x_{i}-\min _{i} x_{i}
$$

En fait, écart-moyen et diamètre moyen sont toujours du même ordre de grandeur.

Désignons par $\varepsilon_{\alpha}(x)$ l'écart-moyen :

$$
\varepsilon_{\alpha}(x)=\left(\frac{1}{n} \sum\left|x_{i}-c\right|^{\alpha}\right)^{1 / \alpha}
$$

où $c$ est le centre.

Alors, on peut démontrer (cf. [Barbut, 1986(b)] la double inégalité :

$$
2 \varepsilon_{\alpha}(x) \geq \delta_{\alpha}(x)>\varepsilon_{\alpha}(x)
$$

Ainsi, de même qu'on dit diamètre, on pourrait considérer que l'écart est le rayon de la distribution.

\subsection{L'INÉGALITÉ TRIANGULAIRE}

Dans les trois cas $(\alpha=1, \alpha=2, \alpha=\infty)$ usuels, on vérifie aisément :

(6)

$$
D_{\alpha}(x+y) \leq D_{\alpha}(x)+D_{\alpha}(y),
$$$$
\alpha \geq 1
$$

- Pour $\alpha=1$

$$
D_{1}(x+y)=\sum_{i} \sum_{j}\left|\left(x_{i}+y_{i}\right)-\left(x_{j}+y_{j}\right)\right|=\sum \sum\left|\left(x_{i}-x_{j}\right)+\left(y_{i}-y_{j}\right)\right|
$$

Or, pour chaque couple $(i, j)$ :

$$
\left|\left(x_{i}-x_{j}\right)+\left(y_{i}-y_{j}\right)\right| \leq \mid\left(x_{i}-x_{j}|+| y_{i}-y_{j} \mid\right.
$$

D'où l'inégalité (6).

- Pour $\alpha=2$, on a :

$$
\begin{aligned}
\left(D_{2}(x+y)\right)^{2} & =\sum_{i} \sum_{j}\left(x_{i}+y_{i}-x_{j}-y_{j}\right)^{2}=\sum_{i} \sum_{j}\left(x_{i}-x_{j}+y_{i}-y_{j}\right)^{2} \\
& =\sum_{i} \sum_{j}\left(x_{i}-x_{j}\right)^{2}+\sum_{i} \sum_{j}\left(y_{i}-y_{j}\right)^{2}+2 \sum \sum\left(x_{i}-x_{j}\right)\left(y_{i}-y_{j}\right) \\
& =\left(D_{2}(x)\right)^{2}+\left(D_{2}(y)\right)^{2}+2 \sum \sum\left(x_{i}-x_{j}\right)\left(y_{i}-y_{j}\right)
\end{aligned}
$$

Mais d'après l'inégalité de Schwarz :

$$
\mid \sum \sum\left(x_{i}-x_{j}\right)\left(y_{i}-y_{j} \mid \leq D_{2}(x) D_{2}(y)\right.
$$

D'où la double inégalité : 


$$
\left|D_{2}(x)-D_{2}(y)\right| \leq D_{2}(x+y) \leq D_{2}(x)+D_{2}(y)
$$

Pour $\alpha=\infty$, soit $i_{0}$ l'indice du maximum des $\left(x_{i}+y_{i}\right)$, et $i_{1}$ celui du minimum. On a :

$$
D_{\infty}(x+y)=x_{i_{0}}+y_{i_{0}}-\left(x_{i_{1}}+y_{i_{1}}\right)=\left(x_{i_{0}}-x_{i_{1}}\right)+\left(y_{i_{0}}-y_{i_{1}}\right)
$$

Or : $\quad x_{i_{0}}-x_{i_{1}} \leq \max _{i} x_{i}-\min _{i} x_{i}=D_{\infty}(x)$

Et de même pour $y$. Finalement :

$$
D_{\infty}(x+y) \leq D_{\infty}(x)+D_{\infty}(y)
$$

Pour démontrer (6) dans le cas général où $\alpha \geq 1$ est réel quelconque, rappelons d'abord que dans un espace de dimension $N$, la norme $\|z\|_{\alpha}$ des vecteurs $z=\left(z_{1}, \cdots, z_{v}, \cdots, z_{N}\right)$ définie par :

$$
\|z\|_{\alpha}=\left(\sum_{v=1}^{N}\left|z_{v}\right|^{\alpha}\right)^{1 / \alpha}
$$

vérifie l'inégalité triangulaire (cf. [Hardy, et alii, 1934]); pour une démonstration géométrique élémentaire, cf. [Barbut, 1986(a)].

Posons : $\quad n_{i j}=x_{i}-x_{j} \quad v_{i j}=y_{i}-y_{j}$

On a, selon la définition (2) du diamètre :

et

$$
\begin{aligned}
& D_{\alpha}(x)=\left(\sum_{1}^{n} \sum_{1}^{n}\left|u_{i j}\right|^{\alpha}\right)^{1 / \alpha} \quad D_{\alpha}(y)=\left(\sum_{1}^{n} \sum_{1}^{n}\left|v_{i j}\right|^{\alpha}\right)^{1 / \alpha} \\
& D_{\alpha}(x+y)=\left(\sum_{1}^{n} \sum_{1}^{n}\left|u_{i j}+v_{i j}\right|^{\alpha}\right)^{1 / \alpha}
\end{aligned}
$$

Posons ensuite : $\quad v(i, j)=n(i-1)+j$. Lorsque $i$ et $j$ prennent chacun toutes les valeurs entières de 1 à $n, v(i, j)$ prend une fois et une seule chacune des valeurs entières de 1 à $n^{2}$.

On a donc, dans la dimension $N=n^{2}$ :

$$
D_{\alpha}(x)=\left(\sum_{1}^{n^{2}}\left|u_{v}\right|^{\alpha}\right)^{1 / \alpha}=\|u\|_{\alpha}
$$

De même : $\quad D_{\alpha}(y)=\|v\|_{\alpha} \quad D_{\alpha}(x+y)=\|u+v\|_{\alpha}$

L'inégalité triangulaire pour les normes $\|u+v\|_{\alpha} \leq\|u\|_{\alpha}+\|v\|_{\alpha}$ se ramène donc ici l'inégalité (6).

\subsection{DEUX RELATIONS POUR LE DIAMÈTRE $D_{1}$}

Dans le cas de la métrique de Manhattan $(\alpha=1)$, celle qui est associée à la médiane, le diamètre vérifie quelques relations remarquables. 
En voici une, dont on trouvera une démonstration dans [Barbut, 1986]. Désignons par $x_{R}$ l'ensemble des $x_{i}$ strictement supérieurs à la médiane $\mu$ (les « riches ») et par $x_{P}$ l'ensemble de ceux qui sont inférieurs à $\mu$ (les «pauvres »). Alors on a :

$$
D_{1}(x)=D_{1}\left(x_{R}\right)+D_{1}\left(x_{P}\right)+2\left[\frac{n+1}{2}\right] E_{1}(x)
$$

ou $[a]$ désigne la partie entière de $a$.

En voici une seconde. Supposons ici $n=2 k+1$ et que les indices sont tels que :

$$
x_{1} \leq x_{2} \leq \ldots \leq x_{k+1} \leq \ldots \leq x_{2 k+1}
$$

Alors, en réarrangeant convenablement les termes de la somme double ci-dessous, on trouve :

$$
\text { (7) } \quad \frac{1}{2} D_{1}(x)=\sum_{x_{i}>x_{j}} \sum_{i}\left(x_{i}-x_{j}\right)=2 \sum_{h=1}^{k}\left(x_{k+1+h}-x_{k+1-h}\right) h
$$

Dans le dernier membre de (7), que C. Gini connaissait bien, chacune des différences $\left(x_{k+1+h}-x_{k+1-h}\right)$ est un intervalle interquantile médian, ce qui relie l'idée de diamètre à la troisième idée, celle des intervalles interquantiles, dont on va parler maintenant. Dans les notations du paragraphe 3 , ci-dessous, on aurait $q=\frac{k+h+1}{2 k+1}$ (cf. Figure 7).

\section{TROISIÈME IDÉE : LES INTERVALLES INTERQUANTILES MÉDIANS}

Dans la graphique de la Figure 7, on a en ordonnées le numéro d'ordre de chaque $x_{i}$, ceux-ci étant ordonnés par ordre croissant des indices. Et en abscisses, les $x_{i}$ correspondants.



Figure 7

Ramenées à une échelle allant de 0 à 1 , les ordonnées seront les proportions successives et croissantes d'un pas constant $\frac{1}{2 k+1}$ : 


$$
\frac{1}{2 k+1}<\frac{2}{2 k+1}<\cdots<\frac{k+1-h}{2 k+1}<\cdots<\frac{k+1+h}{2 k+1}<\cdots<1
$$

Ici, $h$ varie de 0 à $k$.

Posons : $\quad q=\frac{k+1+h}{2 k+1}$

Alors l'intervalle $\left(x_{k+1+h}-x_{k+1-h}\right)$ s'écrit $\left(x_{q}-x_{1-q}\right)$.

D'une façon générale, si $F(x)$ est une fonction de répartition continue strictement croissante, tout nombre $q$ tel que $1 \geq q \geq 1 / 2$ correspond par inversion de $F$ un intervalle $I_{q}$ (cf. Figures 8(a) et 8(b)) :

$$
I_{q}=x_{q}-x_{1-q}
$$

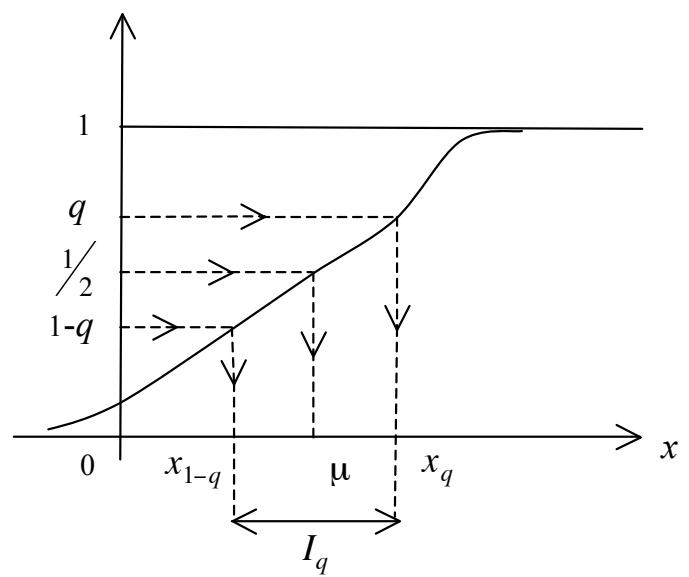

Figure 8(a)

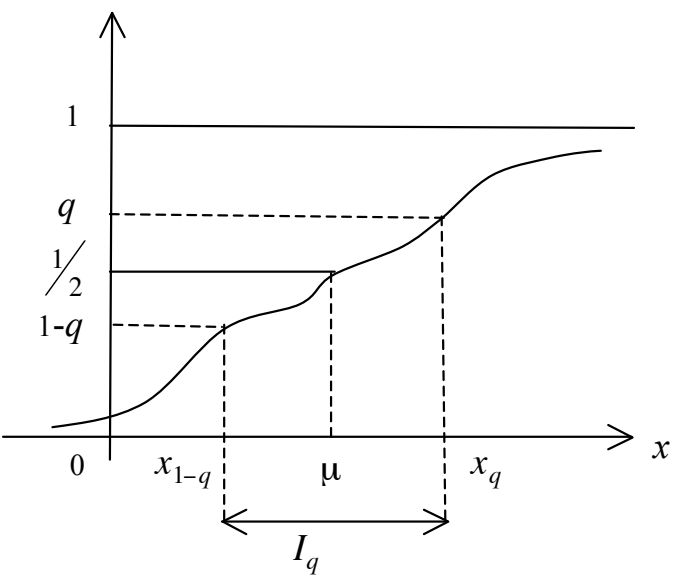

Figure $8(b)$

Il est clair (cf. Figure 8(b)) que moins la distribution de fonction de répartition $F$ est concentrée autour de sa médiane (i.e., plus elle est «étalée »), plus, pour une même valeur de $q$, l'intervalle $I_{q}$ est grand.

L'intervalle $I_{q}$, appelé intervalle interquantile médian, est ainsi un indicateur de la dispersion de la distribution.

Les valeurs usuellement considérées sont :

$-q=\frac{3}{4} \quad$ intervalle interquartile

- $q=\frac{h}{10}, h$ entier allant de 5 à 10 , qui définit les intervalles interdéciles. Mais on utilise aussi les vigintiles, pour lesquels le pas est de $5 \%$.

Cette façon de «mesurer » la dispersion présente deux avantages considérables par rapport aux précédents (écarts et diamètres). 
1. Quelle que soit la variable $X$, qu'elle ait des moments ou non, et en particulier qu'elle ait un moment d'ordre 1 (moyenne) ou non, elle est toujours définie.

Alors que les écarts et les diamètres ne sont définis que si le moment d'ordre 1 existe.

2. Le second avantage, c'est que si, pour une même distribution, on fait varier $q$, l'intervalle $I_{q}$ est une fonction de $q$, monotone croissante (ou non-décroissante) lorsque $q$ va de $1 / 2$ à 1 (cf. Figure 9).

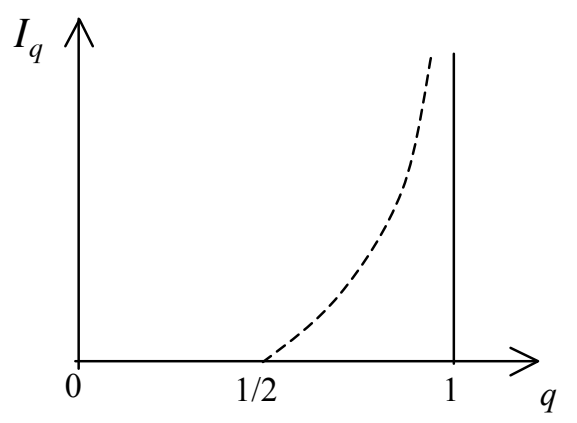

Figure 9

On a ainsi une première définition fonctionnelle de la dispersion ; on en verra d'autres.

Exemples.

- Distribution de Pareto :

$$
F(x)=1-\left(\frac{x_{\circ}+c}{x+c}\right)^{\alpha}, \alpha>0, x \geq x_{0}>-c
$$

Elle n'a de moyenne que pour $\alpha>1$. Mais, quel que soit $\alpha$, pour $F(x)=q$, on a :

$$
q=1-\left(\frac{x_{0}+c}{x+c}\right)^{\alpha}
$$

soit : $\quad(1-q)^{1 / \alpha}=\frac{x_{0}+c}{x+c}$

soit encore : $\quad x=x_{q}=-c+\left(x_{0}+c\right)(1-q)^{-1 / \alpha}$

donc : $\quad x_{1-q}=-c+\left(x_{0}+c\right) q^{-1 / \alpha}$

et : $\quad I_{q}=x_{q}-x_{1-q}=\left[(1-q)^{-1 / \alpha}-q^{-1 / \alpha}\right]$

- Distribution de Cauchy :

$$
F(x)=\frac{1}{2}+\frac{1}{\Pi} \operatorname{Arctg}\left(\frac{x-x_{0}}{a}\right)
$$

Pour celle-ci, aucun moment n'est défini, même pas le premier. 
En procédant comme ci-dessus, on a : $\quad x_{q}=x_{0}+\operatorname{atg}\left(q-\frac{1}{2}\right)$

D'où, compte tenu de : $\quad-\operatorname{tg} \theta=\operatorname{tg}(-\theta)$

$$
I_{q}=x_{q}-x_{1-q}=2 a \operatorname{tg}[\Pi(q-1 / 2)]
$$

- Distribution exponentielle :

$$
F(x)=1-e^{-\frac{\left(x-x_{0}\right)}{\lambda}} \quad \lambda>0, x \geq x_{0}
$$

On obtient : $\quad x_{q}=x_{0}-\lambda \ell_{n}(1-q)$

Et : $\quad I_{q}=\lambda \ell_{n}\left(\frac{q}{1-q}\right)$

Ce qui devrait plaire aux amateurs de $\ll \log$ it $»$.

- Distribution Arc sinus.

Pour $-1 \leq x \leq 1$, on a : $F(x)=\frac{1}{2}+\frac{1}{\Pi} \operatorname{Arcsinus} x$.

D'où (Figure 10(a)) : $f(x)=F^{\prime}(x)=\frac{1}{\Pi} \cdot \frac{1}{\sqrt{1-x^{2}}}$.

Ici la masse est concentrée aux extrémités du support, où la densité est infinie.

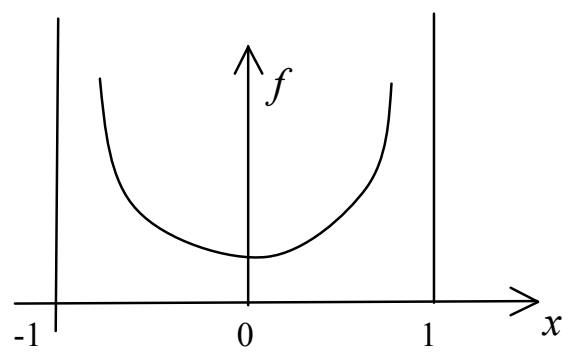

Figure 10(a)

Pour $q>1 / 2$, on a: $q-1 / 2=\frac{1}{\Pi} \operatorname{Arcsin} x_{q}$

D'où : $\quad x_{q}=\sin (\Pi(q-1 / 2)$

Et (Figure 10(b)) : $I_{q}=x_{q}-x_{1-q}=2 \sin (\Pi(q-1 / 2))$ 


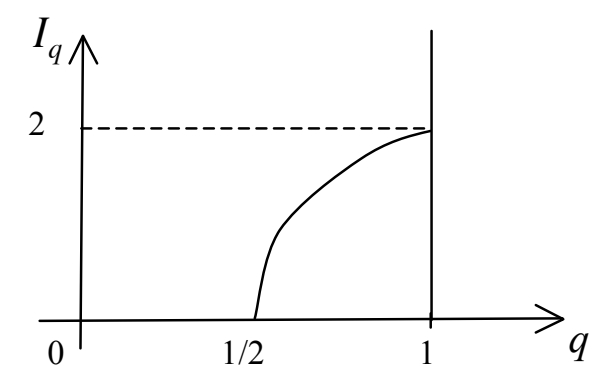

Figure 10(b)

N.B. - On voit sur ces exemples qu'inversement, connaissant l'expression de $I_{q}$ en fonction de $q$, on retrouve aisément celle de $F$ (à un changement d'origine près).

- Dans le cas d'observations empiriques : $x=\left(x_{1}, x_{2}, \cdots, x_{n}\right)$ la fonction de répartition $F$ est une fonction de sauts.

On se ramènera au cas d'une fonction de répartition linéaire par morceaux continue et strictement croissante par interpolation linéaire entre deux sauts successifs.

\section{QUATRIÈME IDÉE : LA FONCTION DE DISPERSION DE PAUL LÉVY}

L'inconvénient majeur de la mesure de la dispersion par un seul nombre tel qu'un écart ou un diamètre, c'est qu'une même valeur de l'indicateur de concentration utilisé peut correspondre à des réalités très différentes.

Exemple classique : une courbe de densité «en cloche» (cf. Figure 11(a)), où la masse se concentre autour d'une valeur centrale peut avoir même variance qu'une courbe « en $\mathrm{U} »$ (cf. Figure 11(b)) où la masse se concentre au contraire aux extrémités de l'intervalle de définition.

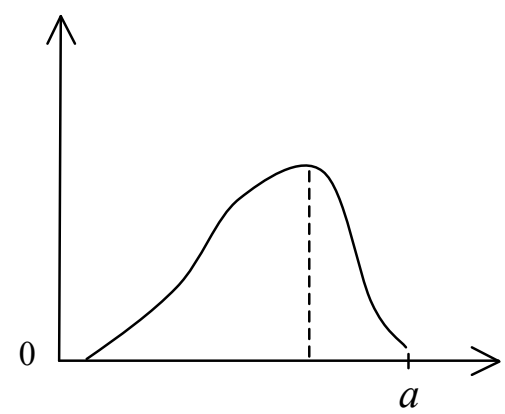

Figure 11(a)

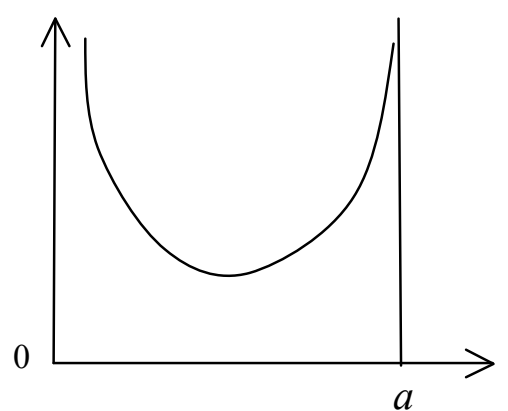

Figure 11(b)

De tels exemples sont faciles à construire. D'où l'intérêt de définitions fonctionnelles de la dispersion qui, elles, prennent en compte toute la distribution, et pas seulement un paramètre de celle-ci. 
On a vu $(\S 3)$ que la considération des quantiles fournit une solution.

L'idée du grand probabiliste Paul Lévy est à première vue assez voisine ; mais on verra qu'en fait elle est notablement différente.

Soit toujours $F$ la fonction de répartition d'une variable $X$. Supposons-la continue et strictement croissante. Soit alors $F$ - l'inverse de $F$.

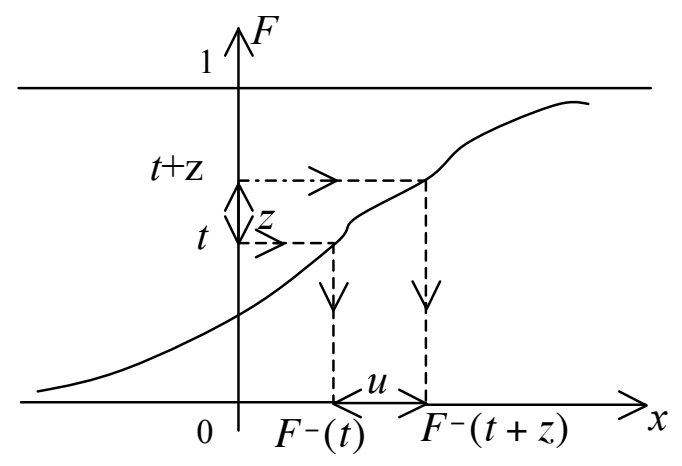

Figure 12

Considérons, sur l'axe des ordonnées, un intervalle $(t, t+z)$ de longueur fixée $z$, et déplaçons-le en faisant varier $t$ de 0 à $1-z$.

Pour chaque valeur de $t$, il y a un intervalle inverse $u$ (cf. Figure 12) :

$$
u=F^{-}(t+z)-F^{-}(t)
$$

Paul Lévy appelle fonction de dispersion de la variable $X$, et note $\omega(z)$, le minimum de $u$ lorsque $t$ varie de 0 à $1-z$ :

$$
\omega(z)=\min _{t}\left[F^{-}(t+z)-F^{-}(t)\right], \quad 0 \leq z \leq 1
$$

Autrement dit, $\varpi(z)$ est le plus petit intervalle de variation de $X$ qui contienne la masse $z$.

Dualement, Paul Lévy définit la fonction de concentration de la variable $X$, soit $Q(y)$ dans ses notations par (cf. Figure 13) :

$$
Q(y)=\max _{x}[F(x+y)-F(x)] \quad y \geq 0
$$

C'est le maximum de la masse qui puisse être contenue dans un intervalle de longueur $y$.

En particulier, $Q(0)$ et le plus grand saut de $F ; Q(0)=0$ ssi $F$ est continue.

Les fonctions de dispersion et de concentration, dont les définitions sont duales, sont inverses l'une de l'autre :

$$
Q=\omega^{-} \quad \text { et } \quad \omega=Q^{-}
$$






Figure 13

N.B. Les définitions des fonctions $\omega$ et $Q$ données ici sont sans problème lorsque la fonction de répartition $F$ est continue et strictement croissante. Dans les autres cas, il y a quelques précautions d'ordre technique à prendre. Nous ne les abordons pas, l'essentiel ici étant que l'idée soit bien comprise.

Revenons à l'exemple d'une distribution «en cloche » comparée à une distribution «en $U$ »; leurs fonctions de concentration n'ont pas du tout la même allure (cf. Figures 14(a) et 14(b) respectivement).

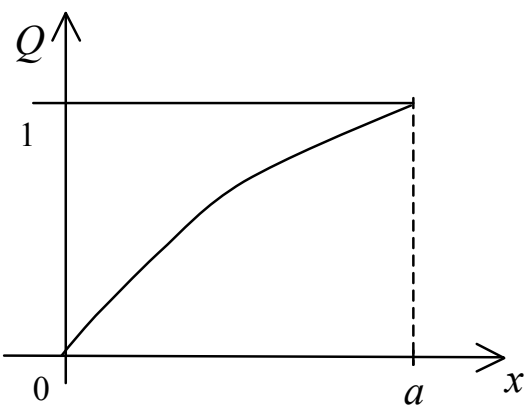

Figure 14(a)



Figure 14(b)

Comme dans le cas des quantiles, on peut se poser la question de l'inversion. Mais là, les choses sont bien différentes.

On a vu dans le paragraphe précédent que la donnée de l'intervalle interquartile médian $I_{q}$ en fonction de $q$ équivalait, à une translation près, à celle de la fonction de répartition.

Par contre, à une fonction de concentration $Q(y)$ donnée, correspondent :

- dans le cas «en cloche», une infinité, dépendant d'une fonction arbitraire, de fonctions de distributions « en cloche » ayant pu l'engendrer ;

- dans le cas « en $U$ », deux distributions au plus susceptibles de l'avoir engendrée.

Ce qui met bien en évidence la différence profonde entre ces deux types de distributions (unimodale ou bi-modale). Au sujet de cette inversion, on peut voir [Barbut, 2002]. 
La fonction de concentration de Lévy satisfait à deux inégalités remarquables, du type « inégalité triangulaire ».

- Si $X$ et $Y$ sont deux variables indépendantes en probabilité, désignons par $Q_{X}$ et $Q_{Y}$ respectivement leurs fonctions de concentration, et par $Q_{X+Y}$ celle de leur somme.

Alors on a uniformément : $\forall t \geq 0, Q_{X+Y}(t) \leq \min \left[Q_{X}(t), Q_{Y}(t)\right]$. Le second membre de cette inégalité est évidemment inférieur à $Q_{X}(t)+Q_{Y}(t)$.

- Si $Q$ est fonction de concentration d'une variable $X$ donnée, elle satisfait à l'inégalité : $\forall x \geq 0, \quad \forall y \geq 0, \quad Q(x+y) \leq Q(x)+Q(y)$.

Cette inégalité, à bien distinguer de celle qui définit la convexité d'une fonction, est d'ailleurs condition suffisante pour que la fonction de répartition d'une variable positive soit une fonction de concentration.

Sur ces deux points, on pourra consulter [Lévy, 1937] ou [Barbut, 2002].

\section{CINQUIÈME IDÉE : LES « SURVIES »}

\subsection{UNE DISPERSION UNILATÉRALE}

En démographie, l'une des variables $X$ beaucoup étudiées est l'âge auquel on meurt, c'est-à-dire la durée de la vie.

Soit un âge $x$, et $m(x)$ la durée moyenne des vies supérieures à $x$. La différence :

$$
s(x)=m(x)-x
$$

est la survie moyenne à l'âge $x$.

On peut d'ailleurs remplacer la durée moyenne par la durée médiane $\mu(x)$ des vies supérieures à $x$. On obtient ainsi comme survie médiane, que les démographes appellent souvent survie probable :

$$
\sigma(x)=\mu(x)-x
$$

Certains statisticiens (W. Lexis, E.J. Gumbel) calculaient les fonctions $s$ et $\sigma$ à partir d'un âge $x$ particulier, celui où la mortalité est maximum, le mode de la distribution dénommé vie normale; si on le note $x_{0}$, on obtient ainsi :

$$
s\left(x_{0}\right)=m\left(x_{0}\right)-x_{0} \quad \sigma\left(x_{0}\right)=\mu\left(x_{0}\right)-x_{0}
$$

Si l'on considère (à tort, comme on l'a souligné ci-dessus $\S 1.3$ ) la vie normale $x_{0}$ comme valeur centrale de la distribution des durées de vie, les deux normes $s\left(x_{0}\right)$ et $\sigma\left(x_{0}\right)$ peuvent être considérées comme des mesures unilatérales de la dispersion à partir de l'âge modal («normal ») $x_{0}$.

C'est ainsi que Gumbel [1938] avait relevé qu'aux États-Unis et au début du $\mathrm{XX}^{\mathrm{e}}$ siècle, la dispersion ainsi calculée des survies était beaucoup plus grande pour les noirs que pour les blancs, alors que ces derniers avaient une «vie normale » très supérieure à celle des noirs. 
Mais on peut plus généralement considérer les fonctions $s(x)$ et $\sigma(x)$ pour chaque valeur possible de la variable $x$. On obtient ainsi deux expressions fonctionnelles de la dispersion pour les valeurs supérieures à $x$.

Ces expressions ont d'ailleurs un sens quelle que soit la variable $X$ en question. Ce sens est particulièrement clair pour les distributions de revenus ou de richesses (cf. $\S 6.2$ ci-dessous).

\subsection{EXEMPLES THÉORIQUES}

Dans ce paragraphe, $P(x)$ désigne toujours la proportion $\operatorname{Pr}(X>x)$ des valeurs de la variable supérieure à $x$; dans certains cas, $P(x)$ peut être interprété comme la probabilité pour que $X$ soit supérieur à $x$.

- Distribution de Pareto: $P(x)=\left(\frac{x_{0}+c}{x+c}\right)^{\alpha}, \quad x \geq x_{0}>-c \quad \alpha>0$

$\mathrm{s}(x)$ n'est définie que pour $\alpha>1$, et vaut : $s(x)=\frac{x+c}{\alpha-1}$

$\sigma(x)$ est défini pour tout $\alpha>0$, et vaut : $\sigma(x)=\left(2^{1 / \alpha}-1\right)(x+c)$.

Dans les deux cas, il s'agit de fonctions linéaires, monotones croissantes de $x$. La dispersion augmente avec $x$; elle tend vers l'infini lorsque $x$ augmente indéfiniment.

- Distribution exponentielle : $P(x)=e^{-\frac{\left(x-x_{0}\right)}{\lambda}} \quad x \geq x_{0} \quad \lambda>0$

$s$ et $\sigma$ sont toujours définies, et l'on a : $s(x)=\lambda \quad \sigma(x)=\lambda \ell_{n}(2)$

Ici, $\ell_{n}$ désigne le logarithme népérien.

La dispersion est constante ; on remarquera d'ailleurs que $\lambda$ est aussi l'écart-type de la distribution.

- Une distribution « sigmoïde » $: P(x)=e^{-\frac{x^{2}}{2 S^{2}}}, \quad x \geq 0$.

On obtient : $s(x)=e^{\frac{x^{2}}{2 S^{2}}} G\left(\frac{x}{S}\right) \sqrt{2 \Pi}$, où $G$ est la distribution de Laplace-Gauss : $\sigma(x)=\left(x^{2}+2 S^{2} \ell(2)\right)^{1 / 2}-x$

Chacune d'entre elles est fonction monotone décroissante de $x$, et tend vers 0 lorsque $x$ augmente indéfiniment. Plus $x$ est grand, moins il y a de dispersion.

- Une loi de mortalité : la loi de Gompertz.

La loi de Gompertz est très fréquemment utilisée pour représenter à partir d'un certain âge (40 ans par exemple) et sauf pour les très grands âges (au-delà de 90-95 ans) les courbes de mortalité des démographes et des actuaires. Elle dépend de deux paramètres $\lambda$ et $v$ positifs et $a$ pour expression :

$$
P(x)=e^{1 / \lambda} e^{-u(x)} \quad \text { avec } \quad u(x)=\frac{1}{\lambda} e^{v x}, \quad \lambda, \nu>0
$$


$v$ est le taux (ou «quotient») de mortalité à l'âge de mortalité maximum (la « vie normale ») et $\lambda$ le rapport de ce taux au taux de mortalité à l'origine choisie pour $x$.

On démontre que (cf. par exemple, [Barbut, 2009]) :

$$
s(x)=\frac{1}{v} \Phi[u(x)] \quad \text { où } \quad \Phi(z)=e^{z} \int_{z}^{\infty} \frac{e^{-t}}{t} d t, \quad z>0
$$

L'intégrale intervenant dans l'expression de $\Phi$ est parfois appelée le «logarithme intégral ».

Quant à la survie probable, son expression est :

$$
\sigma(x)=\frac{1}{v} \ell_{n}\left(e^{v x}+\lambda \ell_{n} 2\right)-x
$$

L'une et l'autre sont fonctions monotones décroissantes de $x$, tendant vers zéro lorsque $x$ augmente indéfiniment.

Mais il semble qu'aux très grands âges, $s$ et $\sigma$ cessent de décroître et restent supérieurs à une borne strictement positive, comme c'est le cas pour la distribution exponentielle.

\subsection{INVERSION DE LA SURVIE MOYENNE}

Inversement et sous des conditions assez générales, si l'on connaît l'expression $s(x)$ de la survie moyenne, on peut retrouver $P(x)$; le comportement est donc ici analogue à celui des intervalles interquantiles (cf. $\S 3$ ) et non à celui de la fonction de concentration de Lévy (cf. § 4).

Supposons que $P(x)$ est dérivable, de dérivée $P^{\prime}=-f(x)$, où $f$ est la densité de la distribution. Et soit $s(x)$ donnée, continue, strictement positive et dérivable, avec $s^{\prime} \geq-1$.

On a par définition : $m(x)=\frac{1}{P(x)} \int_{x}^{\infty} t f(t) d t$

D'où : $s(x)=\left(\frac{1}{P(x)} \int_{x}^{\infty} t f(t) d t\right)-x$

D'où : $s(x) P(x)+x P(x)=\int_{x}^{\infty} t f(t) d t$

Soit, en dérivant : $s^{\prime} P+s P^{\prime}+P+x P^{\prime}=-x f(x)=x P^{\prime}$

Après simplification, il vient donc :

$$
\frac{P^{\prime}}{P}=-\left(\frac{s^{\prime}}{s}+\frac{1}{s}\right)
$$

Comme $s^{\prime} \geq-1$, on a bien, comme il se doit, $P^{\prime} \leq 0$.

Désignons par $S(x)$ une primitive de $\frac{1}{S(x)}: S^{\prime}(x)=\frac{1}{S(x)}$.

Par intégration de (8) on a : $\ell_{n}(P)=-\ell_{n}(s)-S+c^{\text {te }}$ 
D'où : $\quad P(x)=K \frac{1}{S(x)} e^{-S(x)}=K S^{\prime}(x) e^{-S(x)}=K\left(e^{-S(x)}\right)^{\prime}$

$P(x)$ est donc bien déterminé à la constante multiplicative $K$ près.

\section{SIXIÈME IDÉE : MESURER L’INÉGALITÉ PAR DES FONCTIONS}

De même que la dispersion, l'inégalité est susceptible de mesures fonctionnelles, bien plus efficaces que les simples indices ou coefficients numériques de l'inégalité, qui ont été évoqués au $§ 1.4$ ci-dessus.

Nous en présentons sommairement deux, l'un classique, l'autre très méconnu.

\subsection{LES FONCTIONS DE CONCENTRATION DE LORENZ ET GINI}

Elles concernent l'inégalité d'une distribution d'une variable X par rapport à une autre distribution de la même variable (pour une initiation détaillée, cf. [Barbut 1998]).

Prenons l'exemple usuel où $X$ est le revenu distribué dans une population donnée. La proportion de la population ayant un revenu supérieur à $x$ est notée $P(x)$ :

$$
\operatorname{Pr}(X>x)=P(x)
$$

Notons de même $Q(x)$ la proportion du revenu total distribué affectée à ceux qui ont un revenu supérieur à $x$.

$P(x)$ est évidemment fonction monotone non croissante de $x$. Supposons-la, dans le cas de la distribution théorique de $X$ continue, strictement décroissante. Alors à chaque valeur de $P$ correspond une valeur $x$, soit $x(P)$, bien déterminée de $X$ et par conséquent une valeur bien déterminée de $Q ; Q$ est ainsi fonction de la «variable » $P$, comprise entre 0 et 1 :

$$
Q(x(P))=Q[P] \quad 0 \leq P \leq 1
$$

La fonction $Q[P]$ est par définition la fonction de concentration de la distribution $Q$ par rapport à la distribution $P$.

Exemple : $X$ obéit à une loi de Pareto simple :

(9) $\quad 1-F(x)=P(x)=\left(\frac{x_{0}}{x}\right)^{\alpha} \quad x \geq x_{0} \geq 0, \quad \alpha>1$

D'où : $d F(x)=-d P(x)=\frac{\alpha x_{0}^{\alpha}}{x^{\alpha+1}}$

Le revenu moyen distribué est :

$$
m_{0}=\int_{x_{0}}^{\infty} t d F(x)=\alpha x_{0}^{\alpha} \int_{x_{0}}^{\infty} \frac{d t}{t^{\alpha}}=\frac{\alpha}{\alpha-1} x_{0}
$$

La partie de ce revenu détenue par ceux qui ont un revenu supérieur à $x$ est : 


$$
R(x)=\int_{x}^{\infty} t d F(t)=\alpha x_{0}^{\alpha} \int_{x}^{\infty} \frac{d t}{t^{\alpha}}=\frac{\alpha}{\alpha-1} \cdot \frac{x_{0}^{\alpha}}{x^{\alpha-1}}
$$

D'où pour la proportion $Q(x)$ :

$$
Q(x)=\frac{R(x)}{m_{0}}=\left(\frac{x_{0}}{x}\right)^{\alpha-1}
$$

Or, d'après (9) :

$$
\frac{x_{0}}{x}=P^{1 / \alpha}
$$

Donc, finalement, pour $Q$ :

$$
Q[P]=P^{\frac{\alpha-1}{\alpha}}
$$

La Figure (15) est celle de la courbe de concentration (de Lorenz et Gini) correspondante.

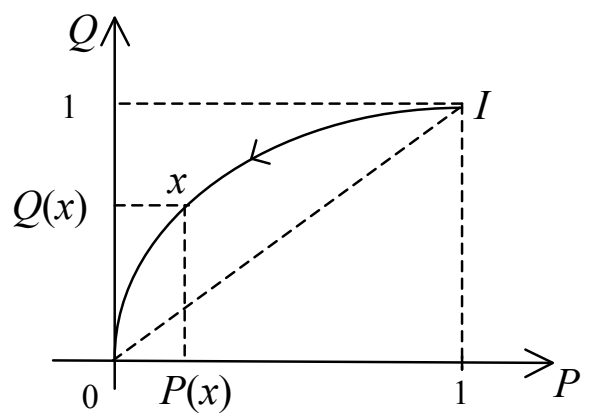

Figure 15

Lorsque $x$ croît de $x_{0}$ à l'infini, elle est parcourue, dans le sens indiqué sur la figure, du point I à l'origine 0 .

N.B. (1) Dans le cas des observations, $P$ et $Q$ sont des distributions en escalier, avec un nombre fini de sauts. On peut interpoler linéairement entre deux sauts successifs pour se ramener aux conditions ci-dessus.

(2) Dans la suite, on omettra les crochets [ ] pour simplifier les notations.

(3) Dans le cas particulier donné en exemple, $m_{0}$ n'est défini que si l'exposant $\alpha$ est supérieur à 1 ; pour $0<\alpha \leq 1, m_{0}$ serait infini et $Q$ n'est plus définie.

D'une façon générale, la fonction de concentration n'existe que si le moment d'ordre 1 (pour $P$ ) est défini.

(4) Rappelons enfin que la courbe de concentration de Lorenz et Gini est toujours convexe, et que, pour toute proportion $P(0<P \leq 1)$ donnée de la population, $Q[P]$ est le maximum de revenu que peut détenir cette proportion.

D'une façon générale, et pourvu que le moment d'ordre 1 existe, on a : 


$$
Q(x)=\frac{1}{m_{0}} \int_{x}^{\infty} t d F(t)=-\frac{1}{m_{0}} \int_{x}^{\infty} t d P(t)
$$

D'où, en différentiant :

$$
d Q=-\frac{1}{m_{0}}(-x d P)=\frac{x}{m_{0}} d P
$$

Et : (11) $\quad \frac{d Q}{d P}=\frac{x}{m_{0}}$

Il en résulte qu'en général la connaissance de la fonction $Q[P]$ entraîne celle de $P$; c'est l'inversibilité de la fonction de concentration.

En effet, supposons $Q[P]$ convexe, dérivable et de dérivée par rapport à $P$ continue. On a :

$$
\frac{d Q}{d P}=h(P)
$$

où $h$ est monotone décroissante lorsque $P$ varie de 0 à 1 ; elle est donc inversible, et l'on a, d'après (11) ci-dessus :

$$
P=h^{-}\left(\frac{x}{m_{0}}\right)
$$

Ce qui définit $P$ à un changement d'échelle près sur la variable $x$.

\subsection{INÉGALITÉ AU SENS DE G. SOREL ET M. FRÉCHET}

Georges Sorel [1897], commentant la loi de Pareto, faisait remarquer que « le sentiment que l'on a de l'inégalité » était bien traduit par le rapport :

$$
\Phi(x)=\frac{m(x)}{x}
$$

où, comme dans le $\S 5$ supra, $m(x)$ est le revenu moyen de ceux qui ont un revenu supérieur à $x$. En effet, plus ce rapport de $m(x)$ à $x$ est grand, plus celui dont le revenu est $x$ ressentira une infériorité par rapport aux plus riches que lui.

Maurice Fréchet [1925] proposait de prendre la fonction $\Phi(x)$ du revenu $x$ comme mesure de l'inégalité.

Il s'agit-là d'une mesure fonctionnelle, d'ailleurs liée à la fonction de survie $s(x)=m(x)-x$ du $§ 5$ par la relation :

$$
\Phi(x)=\frac{m(x)}{x}=\frac{s(x)+x}{x}=1+\frac{s(x)}{x}
$$

Il en résulte immédiatement que, comme $s(x)$, la fonction indicatrice de l'inégalité $\Phi(x)$ est inversible : sa connaissance entraîne celle de la distribution parente $P(x)$, à un facteur près. On a en effet :

$$
s(x)=x(\Phi(x)-1)
$$


Il suffit ensuite d'appliquer le calcul sur $s(x)$ fait au $§ 5.2$ ci-dessus.

\subsection{RELATION ENTRE CES DEUX MESURES DE L'INÉGALITÉ}

On a, par définition de $m(x)$ :

$$
m(x)=\frac{1}{P(x)} \int_{x}^{\infty} t d F(t)
$$

d'où, d'après (10) :

$$
\frac{m(x)}{m_{0}}=\frac{Q(x)}{P(x)}=\operatorname{tg} \theta \quad(\text { cf. Figure 16) }
$$

D'autre part, (11) se traduit géométriquement par :

$$
\frac{x}{m_{0}}=\frac{d Q}{d P}=\operatorname{tg} \varphi
$$

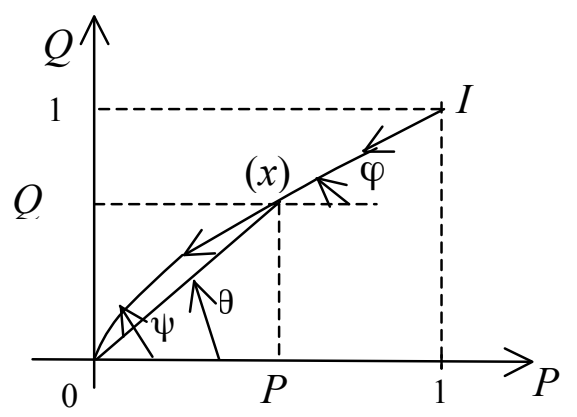

Figure 16

D'où :

$$
\Phi(x)=\frac{Q}{P}: \frac{d Q}{d P}=\frac{d P / P}{d Q / Q}=\frac{\operatorname{tg} \theta}{\operatorname{tg} \varphi}
$$

La relation (12) fournit donc une expression de l'indicatrice de Fréchet au moyen de la fonction de concentration de Lorenz et Gini.

Elle montre en outre qu'en général, si $x$ tend vers l'infini, le point $(x)$ tend vers 0 , et les angles $\theta$ et $\varphi$ tendent tous deux vers $\Psi$, angle de l'axe des abscisses avec la tangente de 0 à la courbe de concentration. Donc, en général :

$$
\lim _{x \rightarrow \infty} \Phi(x)=1
$$

Exceptionnellement, si $\Psi=\frac{\Pi}{2}, \Phi(x)$ devient, lorsque $x$ croît indéfiniment, une forme indéterminée de type $\frac{\infty}{\infty}$. Celle-ci peut éventuellement avoir une limite distincte de 1. 
C'est notamment le cas pour les distributions de Pareto (la limite est $\frac{\alpha}{\alpha-1}$ ) et celle de Galton (la limite est 2, pour la forme normalisée).

N.B. Lorsque le moment d'ordre 1 est infini, on peut remplacer la moyenne par la médiane $\mu(x)$, et prendre comme fonction indicatrice de l'inégalité :

$$
\Psi(x)=\frac{\mu(x)}{x}
$$

Mais ici, l'inversion est moins facile à effectuer.

\section{BIBLIOGRAPHIE}

[Il n'est pas usuel qu'un auteur renvoie pour l'essentiel à ses propres articles. Je le fais ici parce qu'il s'agit de textes dont je suis sûr qu'ils sont à la portée de tous].

ARMATTE M. (1991), « Théorie des erreurs, moyenne et loi normale », Moyenne, milieu, centre, Paris, éd. de l'EHESS, p. 63-84.

BARBUT M. (1986(a)), «Sur deux familles de valeurs centrales généralisant la moyenne arithmétique : moyennes d'ordre $\alpha$, centres d'ordre $\alpha$ », Démographie et sociologie, Paris, Publications de la Sorbonne, p. 131-145.

BARBUT M. (1986(b)), « Diamètres et écarts », Mathématiques et Sciences humaines 93, p. 61-69.

BARBUT M. (1988), « Sur une classe de résumés statistiques : les valeurs centrales », L'à-peu-près, Paris, éd. de l'EHESS, p. 109-141.

BARBUT M. (1991), « Moyennes et valeurs centrales », Moyenne, milieu, centre, Paris, éd. de l'EHESS, p. 135-151.

BARBUT M. (1998), «Une introduction élémentaire à l'analyse mathématique des inégalités », Mathématiques et Sciences humaines 142, p. 27-41. [Repris dans Barbut, 2007, chap. 2].

BARBUT M. (2002), «Une définition fonctionnelle de la dispersion en statistique et en calcul des probabilités : les fonctions de concentration de Paul Lévy », Mathématiques et Sciences humaines 158, p. 31-57. Repris dans Barbut, 2007, chap. 5].

BARBUT M. (2007), «Idéologie et modélisation mathématique », La mesure des inégalités, Genève, Droz, chap. 10, p. 193-205.

BARBUT M. (2009), « Tablas y curvas de mortalitad en el sigle XVIII », Historia de la probabilidad y de la estadistica IV, Universidad de Huelva publicaciones, chap. 9, p. 109-151.

FRÉCHET M. (1925), «Une nouvelle représentation analytique de la répartition des revenus », Actes de la XVIe session de l'Institut international de statistique, Rome, p. 1-3.

GUMBEL E.J. (1937), La durée extrême de la vie humaine, Paris, Hermann.

HARDY G., LiTTLEWOOD J., POLYA G. (1934), Inequalities, Cambridge, Cambridge University Press.

LÉVY P. (1937), Théorie de l'addition des variables aléatoires, Paris, Gauthier-Villars, § 16, p. 43-46, $\S 29$, p. 89-93, § 42, p. 130-133.

SOREL G. (1897), « La loi des revenus », Le devenir social 7, p. 578-607. 